\title{
Some Wgh Inequalities for Univalent Harmonic Analytic Functions
}

\author{
Poonam Sharma \\ Department of Mathematics and Astronomy, University of Lucknow, Lucknow, India \\ E-mail:sharma_poonam@lkouniv.ac.in \\ Received August 2, 2010; revised September 27, 2010; accepted October 2, 2010
}

\begin{abstract}
In this paper, some Wgh inequalities for univalent harmonic analytic functions defined by Wright's generalized hypergeometric (Wgh) functions to be in certain classes are observed and proved. Some consequent results are also discussed.
\end{abstract}

Keywords: Harmonic Functions, Harmonic Starlike Functions, Wright's Generalized Hypergeometric Functions

\section{Introduction and Preliminaries}

Let $u$ and $v$ be real valued harmonic functions in a simply connected domain $D$ in the complex plane $\mathbb{C}$, then a continuous function $f=u+i v$ is called a complex valued harmonic map in $D$. Clunie and SheilSmall [1] introduced a class $S H$ of complex valued harmonic maps $f$ which are univalent and sense-preserving in the open unit disk $\Delta=\{z: z \in \mathbb{C},|z|<1\}$ and assume a normalized representation $h+\bar{g}$ where

$$
h(z)=\sum_{n=1}^{\infty} h_{n} z^{n}, h_{1}=1, g(z)=\sum_{n=1}^{\infty} g_{n} z^{n},\left|g_{1}\right|<1
$$

are analytic and univalent in $\Delta$. Let $S H^{*}(\alpha)$ denotes the class of maps $f=h+\bar{g} \in S H$ satisfying the condition

$$
\frac{\partial}{\partial \theta}\left(\arg \left(f\left(r e^{i \theta}\right)\right)\right)=\operatorname{Im}\left\{\frac{\frac{\partial}{\partial \theta} f\left(r e^{i \theta}\right)}{f\left(r e^{i \theta}\right)}\right\}=\operatorname{Re}\left\{\frac{z f^{\prime}(z)}{f(z)}\right\}>\alpha
$$

for $z=r e^{i \theta}, 0 \leq r<1,0 \leq \theta<2 \pi$ and $0 \leq \alpha<1$, where $z f^{\prime}(z)=z h^{\prime}(z)-\overline{z g^{\prime}(z)}$.

Denote by TSH the subclass of function $f=h+\bar{g} \in S H$ such that

$$
h(z)=z-\sum_{n=2}^{\infty}\left|h_{n}\right| z^{n}, g(z)=\sum_{n=1}^{\infty}\left|g_{n}\right| z^{n} .
$$

Also denote $\operatorname{THS}^{*}(\alpha)=S H^{*}(\alpha) \bigcap T S H$. [2]:

We have following result from the work of Jahangiri
Lemma 1. Let $f=h+\bar{g} \in S H, h(z)$ and $g(z)$ are given by (1), satisfies

$$
\sum_{n=1}^{\infty} n\left\{\left|h_{n}\right|+\left|g_{n}\right|\right\}-\alpha \sum_{n=0}^{\infty}\left\{\left|h_{n+1}\right|-\left|g_{n+1}\right|\right\} \leq 2(1-\alpha)
$$

then $f$ is sense preserving, harmonic univalent in $\Delta$ and $f \in S H^{*}(\alpha)$. Furthermore, $f \in \operatorname{TSH}^{*}(\alpha)$ if and only if (2) holds.

For some $k \in \mathbb{N}=\{1,2,3, \cdots\}$, corresponding to $h(z)$ and $g(z)$ defined in (1), let

$$
f_{k}(z)=h_{k}(z)+\bar{g}_{k}(z) \in S H
$$

where for $z \in \Delta$,

$$
\begin{aligned}
& h_{k}(z)=h(z) * \frac{z}{\left(1-z^{k}\right)}=\sum_{n=0}^{\infty} h_{n k+1} z^{n k+1} \\
& g_{k}(z)=g(z) * \frac{z}{\left(1-z^{k}\right)}=\sum_{n=0}^{\infty} g_{n k+1} z^{n k+1}
\end{aligned}
$$

'*' stands for convolution. Since

$$
\sum_{n=1}^{\infty} h_{n} z^{n}=\sum_{n=0}^{\infty} h_{n k+1} z^{n k+1}+\sum_{m=1}^{k-1} \sum_{n=0}^{\infty} h_{n k+m+1} z^{n k+m+1}
$$

$h_{k}(z)$ and $g_{k}(z)$ for some $k \geq 2$ in (3), represent series of missing terms which increase with $k$. Involving $f_{k}(z)$, defined in (3), a class $S H_{k}(\alpha)$ is defined as follows:

Definition 1. A function $f=h+\bar{g} \in S H$ is said to be in the class $\mathrm{SH}_{k}(\alpha)$, if it satisfies the condition

$$
\operatorname{Re}\left\{\frac{z f^{\prime}(z)}{f_{k}(z)}\right\}>\alpha, 0 \leq \alpha<1,
$$


where for some $k \in \mathbb{N}, f_{k}(z)$ is defined by (3). Functions in the class $S_{k}(\alpha)$ are called harmonic starlike functions with respect to k-symmetric points of order $\alpha$.

Note that

$S H_{1}(\alpha)=S H^{*}(\alpha), S H_{2}(\alpha)=S H_{s}(\alpha)$ and $\operatorname{TSH}_{k}(\alpha)=$ $S_{k}(\alpha) \cap T S H, T S H_{s}(\alpha)=S H_{s}(\alpha) \cap T S H$. The class $\mathrm{SH}_{s}(\alpha)$ is studied by Ahuja and Jahangiri in [3] (see also [4]). They also proved following result in [3].

Lemma 2. Let $f=h+\bar{g} \in S H, h(z)$ and $g(z)$ are given by (1), satisfies

$$
\sum_{n=1}^{\infty} n\left\{\left|h_{n}\right|+\left|g_{n}\right|\right\}-\alpha \sum_{n=0}^{\infty}\left\{\left|h_{2 n+1}\right|-\left|g_{2 n+1}\right|\right\} \leq 2(1-\alpha)
$$

then $f$ is sense preserving, harmonic univalent in $\Delta$ and $f \in S_{s}(\alpha)$. Furthermore, $f \in \operatorname{TSH}_{s}(\alpha)$ if and only if (7) holds.

Shaqsi and Darus in $[5,6]$ proved that for $0 \leq \alpha<1$, $k \in \mathbb{N}$ if $f \in S H_{k}(\alpha)$, then $f_{k} \in S H^{*}(\alpha)$ and proved following result.

Lemma 3. Let $f=h+\bar{g} \in S H, h(z)$ and $g(z)$ are given by (1), satisfies for some $k \in \mathbb{N}$,

$$
\sum_{n=1}^{\infty} n\left\{\left|h_{n}\right|+\left|g_{n}\right|\right\}-\alpha \sum_{n=0}^{\infty}\left\{\left|h_{n k+1}\right|-\left|g_{n k+1}\right|\right\} \leq 2(1-\alpha)
$$

then $f$ is sense preserving, harmonic univalent in $\Delta$ and $f \in S_{k}(\alpha)$. Furthermore, $f \in \operatorname{TSH}_{k}(\alpha)$ if and only if (8) holds.

Obviously Inequality (8) is a generalized inequality ensuring $f$ to be in classes $S H^{*}(\alpha)$ and $S H_{s}(\alpha)$ for $k=1$ and $k=2$ respectively. We see that if inequality (8) holds, inequality (2) must hold for any $0 \leq \alpha<1$ and for $\alpha=0$ both are same. Hence, inequality (8) for $k \in \mathbb{N}$ and $0 \leq \alpha<1$, ensures that $f \in S H^{*}(\alpha)$ and thus it is used in this study.

If $g(z)=0, z \in \Delta$, we denote $S H_{k}(\alpha)=S_{k}(\alpha)$ which is studied by Wang et al. [7] for $\varphi(z)=\frac{(1+(1-2 \alpha) z)}{(1-z)}$ in the respective class. The class $S_{2}(0)=S_{s}^{*}$ is introduced by Sakaguchi [8] whose members satisfy the condition

$$
\operatorname{Re}\left\{\frac{z h^{\prime}(z)}{h_{2}(z)}\right\}>0, z \in \Delta
$$

where

$$
h_{2}(z)=\frac{h(z)-h(-z)}{2} .
$$

Connectivity of hypergeometric functions with harmonic functions is seen through some of the recent papers [9-11]. Specially involvement of Wright's generalized hypergeometric (Wgh) functions is studied in [12-23]. Some Wgh inequalities for starlike and convex classes have already been obtained in [21,23] for certain harmonic functions.
The Wright's generalized hypergeometric (Wgh) function [24,25], for positive real numbers $a_{i}, A_{i}(i=1,2, \cdots, p)$ and $b_{i}, B_{i}(i=1,2, \cdots, q)$ with $1+\sum_{i=1}^{q} B_{i}-\sum_{i=1}^{p} A_{i} \geq 0$, is defined by

$$
\begin{aligned}
& p \Psi q\left[\begin{array}{l}
\left(a_{1}, A_{1}\right),\left(a_{2}, A_{2}\right), \cdots\left(a_{p}, A_{p}\right) \\
\left(b_{1}, B_{1}\right),\left(b_{2}, B_{2}\right), \cdots\left(b_{p}, B_{p}\right)
\end{array} ;\right]= \\
& p \Psi q\left[\begin{array}{l}
\left(a_{i}, A_{i}\right) \\
\left(b_{i}, B_{i}\right)
\end{array} ;\right]=\sum_{n=1}^{\infty} \frac{\prod_{i=1}^{p} \Gamma\left(a_{i}+n A_{i}\right) z^{n}}{\prod_{i=1}^{q} \Gamma\left(b_{i}+n B_{i}\right) n !}, z \in \mathbb{C}
\end{aligned}
$$

Referring to [26], the series in (9) is absolutely convergent $\forall z \in \mathbb{C}$ if $1+\sum_{i=1}^{q} B_{i}-\sum_{i=1}^{p} A_{i}>0$ and if $1+\sum_{i=1}^{q} B_{i}-\sum_{i=1}^{p} A_{i}=0$, it is absolutely convergent for $|z|<\frac{\prod_{i=1}^{q} B_{i}^{B_{i}}}{\prod_{i=1}^{p} A_{i}^{A_{i}}}$ and for $|z|=\frac{\prod_{i=1}^{q} B_{i}^{B_{i}}}{\prod_{i=1}^{p} A_{i}^{A_{i}}}$, $\sum_{i=1}^{q} b_{i}-\sum_{i=1}^{p} a_{i}+\frac{p-q}{2}>\frac{1}{2}$.

Involving Wgh functions as defined in (9), we consider a univalent, harmonic function $W(z)$ of the form:

$$
W(z)=H(z)+\overline{G(z)} \in S H
$$

where

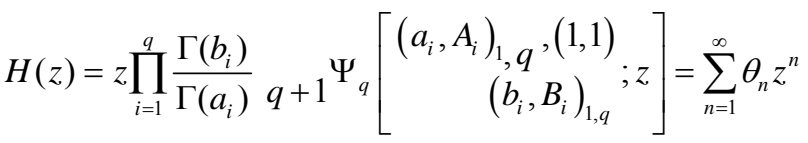

$$
\begin{aligned}
& G(z)=\sigma \prod_{i=1}^{s} \frac{\Gamma\left(d_{i}\right)}{\Gamma\left(c_{i}\right)} \underset{s+1}{s} \Psi_{s}\left[\begin{array}{c}
\left(c_{i}, C_{i}\right)_{1, s},(1,1) \\
\left(d_{i}, D_{i}\right)_{1, s}
\end{array} ; z\right] \\
& =\sigma \sum_{n=1}^{\infty} \zeta_{n} z^{n},|\sigma|<1
\end{aligned}
$$

and

$$
\begin{aligned}
& \theta_{n}:=\prod_{i=1}^{q} \frac{\Gamma\left(a_{i}+(n-1) A_{i}\right) \Gamma\left(b_{i}\right)}{\Gamma\left(b_{i}+(n-1) B_{i}\right) \Gamma\left(a_{i}\right)} \\
& \zeta_{n}:=\prod_{i=1}^{q} \frac{\Gamma\left(c_{i}+(n-1) C_{i}\right) \Gamma\left(d_{i}\right)}{\Gamma\left(d_{i}+(n-1) D_{i}\right) \Gamma\left(c_{i}\right)} .
\end{aligned}
$$

Denote for some $j \in \mathbb{N}_{0}=\mathbb{N} \cup\{0\}$ and for any $k \in \mathbb{N}$, 


$$
\begin{gathered}
\Psi_{q}^{j, k}[z]:={ }_{q+1} \Psi_{q}\left[\begin{array}{c}
\left(a_{i}+j k A_{i}, k A_{i}\right)_{1, q},(1+j, 1) \\
\left(b_{i}+j k B_{i}, k B_{i}\right)_{1, q}
\end{array} ; z\right] \\
\Psi_{S}^{j, k}[z]:={ }_{S+1} \Psi_{S}\left[\begin{array}{c}
\left(c_{i}+j k C_{i}, k C_{i}\right)_{1, s},(1+j, 1) \\
\left(d_{i}+j k D_{i}, k D_{i}\right)_{1, s}
\end{array}\right] z
\end{gathered}
$$

It is noted that at $z=1$, corresponding series of $\Psi_{q}^{j, k}[z], \Psi_{s}^{j, k}[z]$ converge absolutely to $\Psi_{s}^{j, k}[1] \equiv \Psi_{s}^{j, k}$, $\Psi_{s}^{j, k}[1] \equiv \Psi_{s}^{j, k}$, respectively if

1) $\sum_{i=1}^{q}\left(B_{i}-A_{i}\right)>0, \sum_{i=1}^{s}\left(D_{i}-C_{i}\right)>0$, or

2) $\sum_{i=1}^{q}\left(B_{i}-A_{i}\right)=0, \sum_{i=1}^{s}\left(D_{i}-C_{i}\right)=0$ and either

$\prod_{i=1}^{q} \frac{B_{i}^{k B_{i}}}{A_{i}^{k A_{i}}}>1, \prod_{i=1}^{s} \frac{D_{i}^{k D_{i}}}{C_{i}^{k C_{i}}}>1$, or $\prod_{i=1}^{q} \frac{B_{i}^{k B_{i}}}{A_{i}^{k A_{i}}}=1, \prod_{i=1}^{s} \frac{D_{i}^{k D_{i}}}{C_{i}^{k C_{i}}}=1$ with

$$
\sum_{i=1}^{q}\left(b_{i}-a_{i}\right)>1+j, \sum_{i=1}^{s}\left(d_{i}-c_{i}\right)>1+j .
$$

Hence, from (13) and (14), we can easily derive following identities for some $j \in \mathbb{N}_{0}$ and $k \in \mathbb{N}$,

$$
\begin{aligned}
& \sum_{n=j}^{\infty}(n+1-j)_{j} \theta_{n k+1}=\prod_{i=1}^{q} \frac{\Gamma\left(b_{i}\right)}{\Gamma\left(a_{i}\right)} \Psi_{q}^{j, k} \\
& \sum_{n=j}^{\infty}(n+1-j)_{j} \zeta_{n k+1}=\prod_{i=1}^{s} \frac{\Gamma\left(d_{i}\right)}{\Gamma\left(c_{i}\right)} \Psi_{s}^{j, k}
\end{aligned}
$$

provided conditions (1) or (2) of (15) hold. The symbol $(\lambda)_{n}$ called Pochhammer symbol for non negative $n$, is defined by

$$
(\lambda)_{n}=\frac{\Gamma(\lambda+n)}{\Gamma(\lambda)}=\lambda(\lambda+1) \cdots(\lambda+n-1) .
$$

The object of this paper is to examine some Wgh inequalities as a necessary and sufficient conditions for univalent harmonic analytic functions associated with certain Wgh functions to be in the function class $S H_{k}(\alpha)$ for some $k \in \mathbb{N}$ and in particular $S H^{*}(\alpha)$ and $\mathrm{SH}_{s}(\alpha)$. Some consequent results and a convolution property are also derived.

\section{Some Wgh Inequalities}

In order to derive Wgh inequalities, we use Lemma 3.

Theorem 1. Let $W(z)=H(z)+\overline{G(z)} \in S H$ be given by (10), if for

$$
\text { 1) } \sum_{i=1}^{q}\left(B_{i}-A_{i}\right)>0, \sum_{i=1}^{s}\left(D_{i}-C_{i}\right)>0 \text {, or }
$$

2) $\sum_{i=1}^{q}\left(B_{i}-A_{i}\right)=0, \sum_{i=1}^{s}\left(D_{i}-C_{i}\right)=0$ and either for some $k \in \mathbb{N}, \prod_{i=1}^{q} \frac{B_{i}^{k B_{i}}}{A_{i}^{k A_{i}}}>1, \prod_{i=1}^{s} \frac{D_{i}^{k D_{i}}}{C_{i}^{k C_{i}}}>1$, or $\prod_{i=1}^{q} \frac{B_{i}^{k B_{i}}}{A_{i}^{k A_{i}}}=1, \quad \prod_{i=1}^{s} \frac{D_{i}^{k D_{i}}}{C_{i}^{k C_{i}}}=1 \quad$ with $\quad \sum_{i=1}^{q}\left(b_{i}-a_{i}\right)>2$, $\sum_{i=1}^{s}\left(d_{i}-c_{i}\right)>2$, and for some $k \in \mathbb{N}$, Wgh inequality

$$
\begin{gathered}
\prod_{i=1}^{q} \frac{\Gamma\left(b_{i}\right)}{\Gamma\left(a_{i}\right)}\left(\Psi_{q}^{0,1}-\alpha \Psi_{q}^{0, k}+\Psi_{q}^{1,1}\right)+ \\
|\sigma| \prod_{i=1}^{s} \frac{\Gamma\left(d_{i}\right)}{\Gamma\left(c_{i}\right)}\left(\Psi_{s}^{0,1}+\alpha \Psi_{s}^{0, k}+\Psi_{s}^{1,1}\right) \leq 2(1-\alpha)
\end{gathered}
$$

holds, then $W(z)$ is sense preserving, harmonic univalent in $\Delta$ and $W(z) \in S H_{k}(\alpha)$.

Furthermore, $\quad W_{1}(z)=z\left(2-\frac{H(z)}{z}\right)+\overline{G(z)} \in \operatorname{TSH}_{k}(\alpha)$ if and only if (18) holds.

Proof. To show $W(z)$ is sense preserving, harmonic univalent in $\Delta$ and $W(z) \in S H_{k}(\alpha)$, we need to show by Lemma 3, that

$$
\begin{gathered}
S_{1}:=\sum_{n=1}^{\infty} n \theta_{n}-\alpha \sum_{n=0}^{\infty} \theta_{n k+1}+ \\
|\sigma|\left[\sum_{n=1}^{\infty} n \zeta_{n}+\alpha \sum_{n=0}^{\infty} \zeta_{n k+1}\right] \leq 2(1-\alpha) .
\end{gathered}
$$

From the given hypothesis and with the use of identities (16) and (17) for $j=0,1$ and for any $k \in \mathbb{N}$, we observe that

$$
\begin{gathered}
S_{1}=\sum_{n=2}^{\infty}(n-1) \theta_{n}+\sum_{n=1}^{\infty} \theta_{n}-\alpha \sum_{n=0}^{\infty} \theta_{n k+1}+ \\
|\sigma|\left[\sum_{n=1}^{\infty}(n-1) \zeta_{n}+\sum_{n=1}^{\infty} \zeta_{n}+\alpha \sum_{n=0}^{\infty} \zeta_{n k+1}\right] \\
=\prod_{i=1}^{q} \frac{\Gamma\left(b_{i}\right)}{\Gamma\left(a_{i}\right)}\left[\Psi_{q}^{1,1}+\Psi_{q}^{0,1}-\alpha \Psi_{q}^{0, k}\right]+ \\
|\sigma| \prod_{i=1}^{s} \frac{\Gamma\left(d_{i}\right)}{\Gamma\left(c_{i}\right)}\left[\Psi_{s}^{1,1}+\Psi_{s}^{0,1}+\alpha \Psi_{s}^{0, k}\right] \leq 2(1-\alpha)
\end{gathered}
$$

if inequality (18) holds. Furthermore, if $W_{1}(z) \in \operatorname{TSH}_{k}(\alpha)$, by Lemma 3, inequality (19) holds and hence (18) holds. This proves Theorem 1 .

Taking $\mathrm{k}=1$, in Theorem 1, we get following result.

Corollary 1. With the same hypothesis of Theorem 1, for $\mathrm{k}=1$ if Wgh inequality 


$$
\begin{aligned}
& \prod_{i=1}^{q} \frac{\Gamma\left(b_{i}\right)}{\Gamma\left(a_{i}\right)}\left((1-\alpha) \Psi_{q}^{0,1}+\Psi_{q}^{1,1}\right)+ \\
& |\sigma| \prod_{i=1}^{s} \frac{\Gamma\left(d_{i}\right)}{\Gamma\left(c_{i}\right)}\left((1+\alpha) \Psi_{s}^{0,1}+\Psi_{s}^{1,1}\right) \leq 2(1-\alpha),
\end{aligned}
$$

holds, then $W(z)$ is sense preserving, harmonic univalent in $\Delta$ and $W(z) \in S H^{*}(\alpha)$. Furthermore,

$W_{1}(z)=z\left(2-\frac{H(z)}{z}\right)+\overline{G(z)} \in \operatorname{TSH}^{*}(\alpha)$ if and only if (20) holds.

Remark 1. Taking $A_{i}=B_{i}(i=1,2, \cdots, q)$ and $C_{i}=D_{i}(i=1,2, \cdots, s)$, the inequality of Corollary 1 coincides with Theorem 3.1 in [23] for $p=1$.

Taking $\mathrm{k}=2$ in Theorem 1, we get following result.

Corollary 2. With the same hypothesis of Theorem 1, for $\mathrm{k}=2$ if Wgh inequality

$$
\begin{gathered}
\prod_{i=1}^{q} \frac{\Gamma\left(b_{i}\right)}{\Gamma\left(a_{i}\right)}\left(\Psi_{q}^{0,1}-\alpha \Psi_{q}^{0,2}+\Psi_{q}^{1,1}\right)+ \\
|\sigma| \prod_{i=1}^{s} \frac{\Gamma\left(d_{i}\right)}{\Gamma\left(c_{i}\right)}\left(\Psi_{s}^{0,1}+\alpha \Psi_{s}^{0,2}+\Psi_{s}^{1,1}\right) \leq 2(1-\alpha)
\end{gathered}
$$

holds, then $W(z)$ is sense preserving, harmonic univalent in $\Delta$ and $W(z) \in S H_{s}(\alpha)$.

Furthermore, $\quad W_{1}(z)=z\left(2-\frac{H(z)}{z}\right)+\overline{G(z)} \in \operatorname{TSH}_{s}(\alpha)$ if and only if (21) holds.

\section{Consequences of Wgh Inequalities}

Involving Mittag-Leffler functions [25]: $E_{B_{1}, b_{1}}^{1,1}(z)=$ ${ }_{1} \Psi_{1}\left[\left({ }_{\left(b_{1}, B_{1}\right)}^{(1,1)} ; z\right], E_{D_{1}, d_{1}}^{1,1}(z)={ }_{1} \Psi_{1}\left[\begin{array}{c}(1,1) \\ \left(d_{1}, D_{1}\right)\end{array} ; z\right]\right.$, for positive real numbers $b_{1}, B_{1}$ and $d_{1}, D_{1}$, we consider a univalent, harmonic function $E(z)$ for $|\sigma|<1$ of the form:

$$
E(z)=z \Gamma\left(b_{1}\right) E_{B_{1}, b_{1}}^{1,1}(z)+\overline{\sigma z \Gamma\left(d_{1}\right) E_{D_{1}, d_{1}}^{1,1}(z)} \in S H
$$

Denote for some $j \in \mathbb{N}_{0}=\mathbb{N} \cup\{0\}$ and $k \in \mathbb{N}$,

$$
\begin{aligned}
& E_{k B_{1}, b_{1}+j k B_{1}}^{1+j}(z)={ }_{1} \Psi_{1}\left[\begin{array}{c}
(1+j, 1) \\
\left(b_{1}+j k B_{1}, k B_{1}\right)
\end{array} ; z\right], \\
& E_{k D_{1}, d_{1}+j k D_{1}}^{1+j}(z)={ }_{1} \Psi_{1}\left[\frac{(1+j, 1)}{\left(d_{1}+j k D_{1}, k D_{1}\right)} ; z\right] .
\end{aligned}
$$

At $z=1$, corresponding series of $E_{k B_{1}, b_{1}+j k B_{1}}^{1+j, 1}(z)$, $E_{k D_{1}, d_{1}+j k D_{1}}^{1+j, 1}(z)$ converge absolutely to

$E_{k B_{1}, b_{1}+j k B_{1}}^{1+j, 1}(1) \equiv E_{k B_{1}, b_{1}+j k B_{1}}^{1+j, 1}, \quad E_{k D_{1}, d_{1}+j k D_{1}}^{1+j,}(1) \equiv E_{k D_{1}, d_{1}+j k D_{1}}^{1+j, 1}$, respectively. Following result can be directly obtained from Theorem 1.
Corollary 3. Let $E(z)$ be defined by (22), if for some $k \in \mathbb{N}$, inequality

$$
\begin{aligned}
& \Gamma\left(b_{1}\right)\left(E_{B_{1}, b_{1}}^{1,1}-\alpha E_{k B_{1}, b_{1}}^{1,1}+E_{B_{1}, b_{1}+B_{1}}^{2,1}\right)+ \\
& |\sigma| \Gamma\left(d_{1}\right)\left(E_{D_{1}, d_{1}}^{1,1}+\alpha E_{k D_{1}, d_{1}}^{1,1}+E_{D_{1}, d_{1}+D_{1}}^{2,1}\right) \leq 2(1-\alpha),
\end{aligned}
$$

holds, then $E(z)$ is sense preserving, harmonic univalent in $\Delta$ and $E(z) \in S H_{k}(\alpha)$. Furthermore,

$$
\begin{aligned}
& E_{1}(z)= \\
& z\left(2-\Gamma\left(b_{1}\right) E_{B_{1}, b_{1}}^{1,1}(z)\right)+\overline{\sigma z \Gamma\left(d_{1}\right) E_{D_{1}, d_{1}}^{1,1}(z)} \in \operatorname{TSH}_{k}(\alpha)
\end{aligned}
$$

if and only if (23) holds.

Results similar to the Corollaries 1 and 2, for $E(z)$ and $E_{1}(z)$ can be obtained by taking $\mathrm{k}=1$ and $\mathrm{k}=2$ respectively in Corollary 3 .

On taking $A_{i}=B_{i}=1, i=1,2,3, \cdots, q$ and $C_{i}=D_{i}=1$, $i=1,2,3, \cdots, s, W(z)$ reduces to

$$
F(z)=z F_{q}\left(\left(\left[a_{i}\right]\right), z\right)+\overline{\sigma z F_{s}\left(\left(\left[c_{i}\right]\right), z\right)} \in S H,
$$

which involve the generalized hypergeometric functions:

$$
\begin{aligned}
& F_{q}\left(\left(\left[a_{i}\right]\right), z\right) \equiv_{q+1} \Psi_{q}\left[\begin{array}{r}
\left(a_{i}, 1\right)_{1, q},(1,1) \\
\left(b_{i}, 1\right)_{1, q} ; z
\end{array}\right], \\
& F_{s}\left(\left(\left[c_{i}\right]\right), z\right) \equiv_{s+1} \Psi_{s}\left[\begin{array}{c}
\left(c_{i}, 1\right)_{1, s}(1,1) \\
\left(d_{i}, 1\right) 1, s
\end{array}\right] ;
\end{aligned}
$$

Also, if $A_{i}=B_{i}=1, i=1,2,3, \cdots, q$ and $C_{i}=D_{i}=1$, $i=1,2,3, \cdots, s$, for some $j \in \mathbb{N}_{0}=\mathbb{N} \cup\{0\}$ and $k \in \mathbb{N}$, we get

$$
\begin{aligned}
& \prod_{i=1}^{q} \frac{\Gamma\left(b_{i}\right)}{\Gamma\left(a_{i}\right)} \Psi_{q}^{j, k}=\Gamma(1+j) F_{q}^{j, k} \prod_{i=1}^{q} \frac{\left(a_{i}\right)_{j k}}{\left(b_{i}\right)_{j k}} \\
& \prod_{i=1}^{s} \frac{\Gamma\left(d_{i}\right)}{\Gamma\left(c_{i}\right)} \Psi_{s}^{j, k}=\Gamma(1+j) F_{s}^{j, k} \prod_{i=1}^{s} \frac{\left(c_{i}\right)_{j k}}{\left(d_{i}\right)_{j k}}
\end{aligned}
$$

where

$$
\begin{gathered}
F_{q}^{j, k}:=F_{q}^{j, k}\left(\left[a_{i}\right]\right) \equiv \sum_{l=0}^{\infty} \prod_{i=1}^{q} \frac{\left(a_{i}+j k\right)_{l k}}{\left(b_{i}+j k\right)_{l k}} \frac{(1+j)_{l}}{l !}, \\
F_{s}^{j, k}:=F_{s}^{j, k}\left(\left[c_{i}\right]\right) \equiv \sum_{l=0}^{\infty} \prod_{i=1}^{s} \frac{\left(c_{i}+j k\right)_{l k}}{\left(d_{i}+j k\right)_{l k}} \frac{(1+j)_{l}}{l !}
\end{gathered}
$$

provided $\sum_{i=1}^{q}\left(b_{i}-a_{i}\right)>1+j, \sum_{i=1}^{s}\left(d_{i}-c_{i}\right)>1+j$.

From Theorem 1, we obtain following result.

Corollary 4. Let $F(z)$ be defined by (24), if for some $k \in \mathbb{N}$ and $\sum_{i=1}^{q}\left(b_{i}-a_{i}\right)>2, \sum_{i=1}^{s}\left(d_{i}-c_{i}\right)>2$, inequality

$$
F_{q}^{0,1}-\alpha F_{q}^{0, k}+F_{q}^{1,1} \prod_{i=1}^{q} \frac{a_{i}}{b_{i}}
$$




$$
|\sigma|\left(F_{s}^{0,1}+\alpha F_{s}^{0, k}+F_{s}^{1,1} \prod_{i=1}^{s} \frac{c_{i}}{d_{i}}\right) \leq 2(1-\alpha)
$$

holds, then $F(z)$ is sense preserving, harmonic univalent in $\Delta$ and $F(z) \in S H_{k}(\alpha)$. Furthermore, $F_{1}(z)=z\left(2-F_{q}\left(\left(\left[a_{i}\right]\right), z\right)\right)+\overline{\sigma z F_{s}\left(\left(\left[c_{i}\right]\right), z\right)} \in \operatorname{TSH}_{k}(\alpha)$ if and only if (25) holds.

Results similar to the Corollaries 1 and 2, for $F(z)$ and $F_{1}(z)$ can be obtained by taking $\mathrm{k}=1$ and $\mathrm{k}=2$ respectively in Corollary 4 .

Further, taking $q=s=2, b_{2}=d_{2}=1$, in Corollary 4, we get following result for a harmonic univalent function defined by Gauss hypergeometric functions.

Corollary 5. Let for positive real values of $a_{1}, a_{2}, b_{1}, c_{1}, c_{2}, d_{1}$ and for $|\sigma|<1$, a harmonic univalent function:

$$
G(z)=z_{2} F_{1}\left(a_{1}, a_{2} ; b_{1} ; z\right)+\overline{\sigma z_{2} F_{1}\left(c_{1}, c_{2} ; d_{1} ; z\right)} \in S H .
$$

If for some $k \in \mathbb{N}$ and $\left(b_{1}-a_{1}-a_{2}\right)>1$, $\left(d_{1}-c_{1}-c_{2}\right)>1$,

inequality

$$
\begin{gathered}
\left(1+\frac{a_{1} a_{2}}{\left(b_{1}-a_{1}-a_{2}-1\right)}\right) F_{2}^{0,1}\left(\left[a_{i}\right]\right)-\alpha F_{2}^{0, k}\left(\left[a_{i}\right]\right) \\
+|\sigma|\left[\left(1+\frac{c_{1} c_{2}}{\left(d_{1}-c_{1}-c_{2}-1\right)}\right) F_{2}^{0,1}\left(\left[c_{i}\right]\right)+\alpha F_{2}^{0, k}\left(\left[c_{i}\right]\right)\right] \\
\leq 2(1-\alpha),
\end{gathered}
$$

holds, then $G(z)$ is sense preserving, harmonic univalent in $\Delta$ and $G(z) \in S H_{k}(\alpha)$. Furthermore, $G_{1}(z)=z\left(2-{ }_{2} F_{1}\left(a_{1}, a_{2} ; b_{1} ; z\right)\right)+\overline{\sigma z_{2} F_{1}\left(c_{1}, c_{2} ; d_{1} ; z\right)} \in \operatorname{TSH}_{k}(\alpha)$ if and only if (26) holds.

Results similar to the Corollaries 1 and 2, for $G(z)$ and $G_{1}(z)$ can be obtained by taking $\mathrm{k}=1$ and $\mathrm{k}=2$ respectively in Corollary 5 .

\section{Convolution Property}

In this section, we obtain a covolution property for functions belonging to the class $\mathrm{SH}_{k}(\alpha)$.

Theorem 2. A function $f=h+\bar{g} \in S H_{k}(\alpha)$ for some $k \in \mathbb{N}$ if and only if

$$
\begin{aligned}
& h(z) *\left[\frac{(\xi+1) z}{(1-z)^{2}}-\frac{(2 \alpha+\xi-1) z}{\left(1-z^{k}\right)}\right]- \\
& \overline{g(z)} *\left[\overline{\left.\frac{(\xi+1) z}{(1-z)^{2}}+\frac{(2 \alpha+\xi-1) z}{\left(1-z^{k}\right)}\right] \neq 0,}\right.
\end{aligned}
$$

$$
|\xi|=1, \xi \neq-1,0<|z|<1
$$

Proof. From the definition of the function class $S H_{k}(\alpha), f \in S H_{k}(\alpha)$, if and only if

$$
\frac{1}{(1-\alpha)}\left\{\frac{z f^{\prime}(z)}{f_{k}(z)}-\alpha\right\} \neq \frac{\xi-1}{\xi+1},
$$

for $|\xi|=1, \xi \neq-1,0<|z|<1$. Hence by simple calculations, we get

$$
(\xi+1)\left[z f^{\prime}(z)-\alpha f_{k}(z)\right]-(\xi-1)(1-\alpha) f_{k}(z) \neq 0 .
$$

Using (3), we get

$$
\begin{aligned}
& (\xi+1)\left[z h^{\prime}(z)-\overline{z g^{\prime}(z)}-\alpha\left\{h(z) * \frac{z}{\left(1-z^{k}\right)}+\overline{g(z) * \frac{z}{\left(1-z^{k}\right)}}\right\}\right] \\
& -(\xi-1)(1-\alpha)\left\{h(z) * \frac{z}{\left(1-z^{k}\right)}+g(z) * \frac{z}{\left(1-z^{k}\right)}\right\} \neq 0
\end{aligned}
$$

which easily derives the result.

Based on Theorem 2, we get that harmonic functions, $W(z)=H(z)+\overline{G(z)}$

$E(z)=z \Gamma\left(b_{1}\right) E_{B_{1}, b_{1}}^{1,1}(z)+\overline{\sigma z \Gamma\left(d_{1}\right) E_{D_{1}, d_{1}}^{1,1}(z)}$, and $F(z)=z F_{q}\left(\left(\left[a_{i}\right]\right), z\right)+\overline{\sigma z F_{s}\left(\left(\left[c_{i}\right]\right), z\right)}$, defined in (10), (22) and (24) respectively belong to the class $S H_{k}(\alpha)$ for some $k \in \mathbb{N}$ if and only if for $|\xi|=1, \xi \neq-1,0<|z|<1$,

$$
\begin{gathered}
H(z) *\left[\frac{(\xi+1) z}{(1-z)^{2}}-\frac{(2 \alpha+\xi-1) z}{\left(1-z^{k}\right)}\right]- \\
\overline{G(z)} *\left[\overline{\left.\frac{(\xi+1) z}{(1-z)^{2}}+\frac{(2 \alpha+\xi-1) z}{\left(1-z^{k}\right)}\right] \neq 0,}\right. \\
z \Gamma\left(b_{1}\right) E_{B_{1}, b_{1}}^{1,1}(z) *\left[\frac{(\xi+1) z}{(1-z)^{2}}-\frac{(2 \alpha+\xi-1) z}{\left(1-z^{k}\right)}\right]- \\
\left.\frac{\sigma z \Gamma\left(d_{1}\right) E_{D_{1}, d_{1}}^{1,1}(z)}{\left(\frac{(\xi+1) z}{(1-z)^{2}}+\frac{(2 \alpha+\xi-1) z}{\left(1-z^{k}\right)}\right] \neq 0}\right]
\end{gathered}
$$

and

$$
\begin{gathered}
z F_{q}\left(\left(\left[a_{i}\right]\right), z\right) *\left[\frac{(\xi+1) z}{(1-z)^{2}}-\frac{(2 \alpha+\xi-1) z}{\left(1-z^{k}\right)}\right]- \\
\overline{\sigma z F_{s}\left(\left(\left[c_{i}\right]\right), z\right)} *\left[\overline{\left.\frac{(\xi+1) z}{(1-z)^{2}}+\frac{(2 \alpha+\xi-1) z}{\left(1-z^{k}\right)}\right] \neq 0}\right.
\end{gathered}
$$

respectively hold. 


\section{References}

[1] J. Clunie and T. Sheil-Small, "Harmonic Univalent Functions," Annales Academiae Scientiarum Fennicae. Series A I. Mathematica, Vol. 9, 1984, pp. 3-26.

[2] J. M. Jahangiri, "Harmonic Functions Starlike in the Unit Disk," Journal of Mathematical Analysis and Applications, Vol. 235, No. 2, 1999, pp. 470-477.

[3] O. P. Ahuja and J. M. Jahangiri, "Sakaguchi-Type Harmonic Univalent Functions," Scientiae Mathematicae Japonicae, Vol. 59, 2004, pp. 239-244.

[4] H. Ö. Guney, "Sakaguchi-Type Harmonic Univalent Functions with Negative Coefficients," International Journal of Contemporary Mathematical Sciences, Vol. 2, No. 10, 2007, pp. 459-463.

[5] M. Al. Shaqsi and M. Darus, "On Subclass of Harmonic Starlike Functions with Respect to K-Symmetric Points," International Mathematical Forum, Vol. 2, No. 57, 2007, pp. 2799-2805.

[6] M. Al. Shaqsi and M. Darus, "On Harmonic Univalent Functions with Respect to K-Symmetric Points," International Journal of Contemporary Mathematical Sciences, Vol. 3, No. 1-4, 2008, pp. 111-118.

[7] Z. G. Wang, C. Y. Gao and S. M. Yuan, "On Certain Subclasses of Close-to-Convex and Quasi-Convex Functions with Respect to K-Symmetric Points," Journal of Mathematical Analysis and Applications, Vol. 322, No. 1, 2006, pp. 97-106.

[8] K. Sakaguchi, "On Certain Univalent Mapping," Mathmatical Society of Japan, Vol. 11, 1959, pp. 72-75.

[9] O. P. Ahuja, "Planar Harmonic Convolution Operators Generated by Hypergeometric Functions," Integral Transforms and Special Functions, Vol. 18, No. 3, 2007, pp. 165-177.

[10] O. P. Ahuja, "Harmonic Starlike and Convexity of Integral Operators Generated by Hypergeometric Series," Integral Transforms and Special Functions, Vol. 20, No. 8, 2009, pp. 629-641.

[11] O. P. Ahuja and H. Silverman, "Inequalities Associating Hypergeomatric Functions with Planer Harmonic Mappings," Journal of Inequalities in Pure and Applied Mathematics, Vol. 5, No. 4, 2004.

[12] M. K. Aouf and J. Dziok, "Distortion and Convolutional Theorems for Operators of Generalized Fractional Calculus Involving Wright Function," Journal of Applied Analysis, Vol. 14, No. 2, 2008, pp. 183-192.

[13] M. K. Aouf and J. Dziok, "Certain Class of Analytic Functions Associated with the Wright Generalized Hypergeometric Function," Journal of Mathematics and Applications, Vol. 30, 2008, pp. 23-32.

[14] J. Dziok and R. K. Raina, "Families of Analytic Func tions Associated with the Wright Generalized Hypergeometric Function," Demonstratio Mathematica, Vol. 37, No. 3, 2004, pp. 533-542.

[15] J. Dziok and R. K. Raina, "Some Results Based on First Order Differential Subordination with the Wright's Generalized Hypergeometric Function," Commentarii Mathematici Universitatis Sancti Pauli, Vol. 58, No. 2, 2009, pp. 87-94.

[16] J. Dziok, R. K. Raina and H. M. Srivastava, "Some Classes of Analytic Functions Associated with Operators on Hilbert Space Involving Wright's Generalized Hypergeometric Function," Proceedings of the Jangjeon Mathematical Society, Vol. 7, 2004, pp. 43-55.

[17] G. Murugusundaramoorthy and R. K. Raina, "On a Subclass of Harmonic Functions Associated with the Wright's Generalized Hypergeometric Functions," Hacettepe Journal of Mathematics and Statistics, Vol. 38, No. 2, 2009, pp. 129-136.

[18] G. Murugusundaramoorthy and K. Vijaya, "A Subclass of Harmonic Functions Associated with Wright's Hypergeometric Functions," Applied Mathematics, Vol. 1, No. 2, 2010, pp. 87-93.

[19] R. K. Raina, "Certain Subclasses of Analytic Functions with Fixed Argument of Coefficients Involving the Wright's Function," Tamsui Oxford Journal of Mathematical Sciences, Vol. 22, No. 1, 2006, pp. 51-59.

[20] R. K. Raina, “On Generalized Wright's Hypergeometric Functions and Fractional Calculus Operators," East Asian Journal of Mathematics, Vol. 21, No. 2, 2005, pp. 191203.

[21] R. K. Raina and P. Sharma, "Harmonic Univalent Functions Associated with Wright's Generalized Hyper- geometric Functions," Integral Transform and Special Functions, Communicated for Publication.

[22] P. Sharma, "A Class of Multivalent Analytic Functions with Fixed Argument of Coefficients Involving Wright's Generalized Hypergeometric Functions," Bulletin of Mathematical Analysis and Applications, Vol. 2, No. 1, 2010, pp. 56-65.

[23] P. Sharma, "Multivalent Harmonic Functions Defined by M-Tuple Integral Operators," Commentationes Mathematicae, Vol. 50, No. 1, 2010, pp. 87-101.

[24] E. M. Wright, "The Asymptotic Expansion of the Generalized Hypergeometric Function," Proceedings London Mathematical Society, Vol. 46, No.1, 1946, pp. 389-408

[25] H. M. Srivastava and H. L. Manocha, "A Treatise on Generating Functions," Halsted Press, Ellis Horwood Limited, Hichester, 1984.

[26] A. A. Kilbas, M. Saigo and J. J. Trujillo, "On the Generalized Wright Function," Fractional Calculus and Applied Analysis, Vol. 5, No. 4, 2002, pp. 437-460. 